\title{
Challenges and Promises of 5G for Smart Grid Teleprotection Applications
}

\author{
Kinan Ghanem \\ Power Networks Demonstration Centre \\ University of Strathclyde \\ Glasgow, United Kingdom \\ kinan.ghanem@strath.ac.uk \\ Rameez Asif \\ Power Networks Demonstration Centre \\ University of Strathclyde \\ Glasgow, United Kingdom \\ rameez.asif@strath.ac.uk
}

\author{
Stephen Ugwuanyi \\ Electrical and Electronic Engineering \\ University of Strathclyde \\ Glasgow, United Kingdom \\ stephen.ugwuanyi@strath.ac.uk \\ James Irvine \\ Electrical and Electronic Engineering \\ University of Strathclyde \\ Glasgow, United Kingdom \\ j.m.irvine@strath.ac.uk
}

\begin{abstract}
This work is based on some ongoing technical projects, research activities, and discussions for applying $5 G$ technology in smart grid critical applications, specifically in teleprotection. The functional and performance requirements of 5G capabilities needed to meet the overall teleprotection essentials in the smart grid will be discussed. Recommendations that cover the main challenges and promises/prospects for 5G in teleprotection will also be presented.
\end{abstract}

Keywords-5G, smart grid, synchronization, security, teleprotection.

\section{INTRODUCTION}

With appropriate management of smart grid applications, many existing problems exist such as the difficulties in analyzing the field measurement data remotely and the increased time needed to process such amount of data along with the existing cost and the cyber security challenges. Beyond that, there is a need for technology which could help improving the efficiency of data processing in remote applications. 5G could be used in different Machine-toMachine (M2M) applications for smart grid due to how they are interrelated [1]. In [2], 5G is identified as the potential for the realization of a truly converged smart grid infrastructure. Similarly, as presented in [3], cellular networks of today such as $4 \mathrm{G}$ are still very relevant for smart grid applications but $5 \mathrm{G}$ will make the attainment of a novel intelligent time-critical smart grid processing and connectivity possible. However, the main value of $5 \mathrm{G}$ compared with the $4 \mathrm{G}$ in terms of massive data rate may not find many direct applications in the smart grid when the required data rates can be met by $4 \mathrm{G}$. Another promising feature of $5 \mathrm{G}$, which is the Ultra-reliable lowlatency Communications (uRLLC) which can be seen only with the 5th Generation (5G) New Radio (NR). It may also find many applications in the M2M connections between the smart grid distributed devices, where the latency that could be achieved from $5 \mathrm{G}$ could open the door for more advanced use cases in protection applications where the guaranteed end to end delay with less than $6 \mathrm{~ms}$ is needed for some applications such as teleportation.

With the advancement in $5 \mathrm{G}$ development, meeting the low latency, high bandwidth and ultra-reliability demands of smart grid networks can be realized through key 5G technologies. Transitioning the core networks of the power grid from the current protocols such as Multi-Protocol Label Switching (MPLS) and 4G to 5G cellular technology will offer the performance requirements of smart grid control commands, alarms and teleprotection services of $\leq 1 \mathrm{~ms}$, and user plane latency corresponding to 32 bytes of data [4]. The

This work was funded under the research programme of the University of Strathclyde's Power Networks Demonstration Centre (PNDC) future standard releases of $5 \mathrm{G}$ will potentially achieve reliability of $10^{-6}$ Block Error Rate (BLER) and latency in the sub-ms range and satisfying a high capacity transport network at lower Operational Cost (OpEx) becomes realistic. These smart grid requirements have been matched with various $5 \mathrm{G}$ services [5].

\section{RELATED WORK}

Smart grid technology has continued to evolve with the development of advanced communication systems for precise load control, distribution automation, and acquisition of timesensitive information that brings the internet of the smart grid into the future. The upgrade of communication infrastructure is a continuous trend within the utility sector in the search for a network solution that achieves good network performance, higher data rate and lower latency at a lower cost while supporting massive connections. The legacy and current evolutions of cellular networks have remained beneficial to the realization of the pervasive large-scale latency-sensitive data processing by utility companies. Emerging technologies such as the Internet of Things (IoT) and 5G are options to effectively measure, monitor, and control grid parameters in all domains, ie. generation, transmission, distribution, and consumption. In the Ericsson forecast of 2019, 5G connectivity was expected to surpass 2.6 billion towards the end of 2025 [6]. Some of these connections are expected to come from $5 \mathrm{G}$ services for the critical applications of the grid at the reliability of $99.99 \%$ with a latency of $1 \mathrm{~ms}$ (theoretically) and data rate of 10 Gbps [7]. These performance indicators represent demanding and important use cases in the energy sectors where bandwidth, latency, and reliability remain open research issues due to the stringent communication requirements between sections of the grid. Wireless technology is presently preferred over the wire type for cost and ease of deployment in difficult terrains [8], but converged solutions of fiber and $5 \mathrm{G}$ will make an improved time-to-market strategy of varied terms in the management of different smart grid generation, transmission and distribution sources as recommended in [2]. 
Table 1 Summary of Related Literature and their contributions

\begin{tabular}{|c|c|c|}
\hline Authors & Year & Reviewed area and contribution \\
\hline Helen et al. [9] & 2020 & $\begin{array}{l}\text { 5G for smart grid and proposed a } \\
\text { proof of concept architecture which } \\
\text { exploits NFVs in } 5 \mathrm{G} \text { to build an } \\
\text { enhanced smart grid network. }\end{array}$ \\
\hline $\begin{array}{l}\text { Seppo et al. } \\
{[10]}\end{array}$ & 2019 & $\begin{array}{l}\text { Comparison of Network Time } \\
\text { Protocol (NTP) performance in } \\
4 \mathrm{G} / 5 \mathrm{G} \text { networks with } 5 \mathrm{G} \text { network } \\
\text { delivering an improved NTP's } \\
\text { performance in the management of } \\
\text { the complex service for critical smart } \\
\text { grid applications. }\end{array}$ \\
\hline $\begin{array}{l}\text { Tesfaye } \\
\text { Bjarne [11] }\end{array}$ & 2019 & $\begin{array}{l}\text { Proposed concepts for using edge } \\
\text { virtualization in } 5 \mathrm{G} \text { for grid } \\
\text { distributed protection functions. The } \\
\text { stochastic analysis of the } 5 \mathrm{G} \text { model, } \\
\text { when compared to the Ethernet-based } \\
\text { IEC } 61850 \text { system, showed a higher } \\
\text { availability of protection functions. }\end{array}$ \\
\hline $\begin{array}{l}\text { Bhargav et al. } \\
\text { [7] }\end{array}$ & 2018 & $\begin{array}{l}\text { Smart grid communication systems } \\
\text { and standards where IoT and } 5 \mathrm{G} \text { were } \\
\text { identified as potential technologies to } \\
\text { transform protection services in smart } \\
\text { grid. }\end{array}$ \\
\hline Garhi et al. [12] & 2017 & $\begin{array}{l}\text { Challenges and opportunities of } \\
\text { existing technology and envisioned } \\
5 \mathrm{G} \text { to mitigate outages and support } \\
\text { more energy sources in the power } \\
\text { grid. }\end{array}$ \\
\hline
\end{tabular}

\section{MAIN APPLICATIONS OF 5G IN SMART GRID}

The main application of $5 \mathrm{G}$ in the context of this paper is for teleprotection services which is a very important feature in 4G and 5G M2M communication standards [13] - [14]. This characteristic could cause significant enhance in the management of the smart grid via reducing the latencies associated with a simultaneous operation during any critical fault in the power network. Considering the main wireless communication requirements for the smart grid to enable any of their critical applications such as teleprotection, several implementation and integration challenges exist. Most wireless technologies used in smart grid applications such as remote monitoring for secondary substation face the following challenges and limitations due to increasing grid complexity [15] - [16]:

- Integration with legacy communication equipment, as some existing hardware has limited capabilities in supporting the latest communication protocols and security features.

- Interoperability between different vendors' equipment along with the life-time of both the power and the communication assets.

- Security challenges, as securing the connection will increase the bandwidth overhead needed for each application and may require more processing time. Packets queuing at any communication gateway may increase the delay time of that and other packets.

- Limitations with available licensed radio frequency (RF) spectrum to power utility networks.
- Lack of backup power for the wireless services provided by mobile network operators.

- Lack of reach and limit of penetration of wireless communication signal in some remote sites, rural and hard to reach areas.

- Latency: The best operational scenario of the smart grid is in the order of few milliseconds for real-time applications. Shared communication resources defeat this aim without QoS and security issues.

- Reliability: The reliability of most grid applications stands in the value of $99.9999 \%$. Unavailability of service must be avoided to fulfil the SLA.

Any of the above limitations and challenges can prevent any sufficient use of wireless communications in a very critical application such as teleprotection, where very high availability along with enough resilience are essential. However, for Wide Area Network (WAN) communication applications such as teleprotections where the transmission comprises both radio access and wide-area core networks, the end-to-end delay with several hops in the radio access networks may not be able to meet the requirements for critical teleprotection applications. Other special areas of application of $5 \mathrm{G}$ for smart grid include:

\section{A. Network Slicing}

Virtualization of network resources such as the Network Slicing (NS) has enabled improved operational efficiency and cost savings in the operation of the smart grid. As evidenced in [17], network slicing can be performed at the access, transport, and core network layers according to the dynamic nature of service requirements. From the techno-economic view of $5 \mathrm{G}$ for teleprotection, NS economic model described in [18] is used to manage the Total Cost of Ownership (TCO) and Return on Investment (ROI) in smart grid projects. Advanced Metering Infrastructure (AMI), distribution automation, UAV based grid inspection and level precision control are the network slicing use cases for 5G. Simplifying utility network into a single resource offering with multiple services enabled by Software Defined Networking (SDN), Networking Function Virtualisation (NFV), etc are some of the viabilities of implementing network slicing. This means saving on Operational Expenditure (OPEX) by sharing infrastructure.

\section{B. Ultra-Reliable Low-Latency Communication (uRLLC)}

The URLLC specification in the 3GPP standard Release 15 is for real-time industrial automation and electricity distribution use cases. The overreaching role of $5 \mathrm{G}$ is focused on matching uRLLC, mMTC and eMBB technologies with smart grid services. The latency insensitive applications in the utility like smart metering, environmental monitoring could easily be provisioned using technologies like NB-IoT and eMTC which is now part of the $5 \mathrm{G}$ design specification. URLLC can be used in new smart grid applications to support and enable many critical applications such as protection automation, interlocking, digital substations, remote control for $\mathrm{HV}$ and Virtual/Augmented Reality (VR/AR) in primary substation environment. The previous use cases were not supported by any wireless technology before the $4 \mathrm{G}$, as the limitations of the bandwidth offered by the previous wireless 
technologies cannot cope with such high demanding applications in terms of bandwidth and latency.

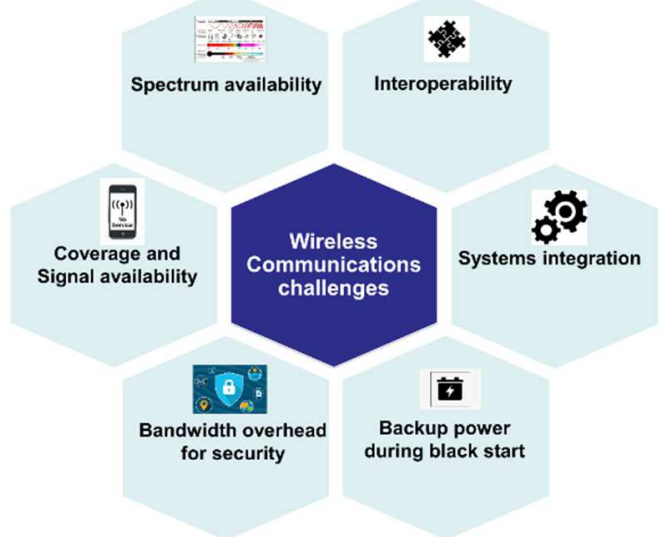

Figure 1 Main Challenges of existing technologies in smart grid applications

\section{TELEPROTECTION REQUIREMENTS FOR SMART GRID}

Teleprotection system is setup to monitor transmission line, prevent any failures or damages and quickly disconnects the faulty part of the power network. To ensure that the power network is fully protected, the communication traffic used to enable the teleprotection functions must be highly reliable, resilient, and trusted. For such communication technology, the main criteria needed for teleprotection application are summarized below:

- Propagation and asymmetrical latency, the main recommendations from the ENA TS 48-6-7 illustrated in table 1 showed that the most stringent propagation and asymmetrical latency requirement is the category 1 which requires less than $(6 \mathrm{~ms})$ and $(0.4 \mathrm{~ms})$ respectively, and both should be satisfied before applying any communication for teleprotection.

- $\quad$ Relay trip time upon power system fault, the results depicted the relay tripping time requirement defined in the National Grid TS 3.24.18 should be satisfied.

- Congested traffic, this should be tested to make sure that the network latency and asymmetrical latency seen by the relays would not be affected even when no priority was assigned to the relay traffic. Moreover, ensuring that the congested traffic will not add further delay on the transmitted traffic between the relays. This is very crucial before any field deployment for any technology (i.e. $5 \mathrm{G}$ for teleprotection).

- Radio path attenuation, this also should be tested for any wireless technology aiming to check whether the network latency and asymmetrical latency would be affected by the radio link and deteriorated by the attenuation or any change in the modulation (as most recent technologies support adaptive modulation techniques).

- Network path switching test, this is to ensure that the network switchover time is always less than recommended threshold in the industrial standards and there is no relay mal-operation (i.e. not falsely tripping the circuit) during path switching throughout the tests. In mobile networks, switching the path and relocation should be performed without any possibility for maloperation.
- Cyber security test, this is to guarantee that any vulnerability from security for $5 \mathrm{G}$ should not cause any relay mal-operation.

Teleprotection applications for Smart grid have the strictest communication requirements. The communication latency for some categories is less than $6 \mathrm{~ms}$, with a reliability of 99.999. This could make it difficult for many existing communication technologies to comply with. Current teleprotections applications rely on wired communication technologies, namely communication via fibre optic, that is widely used for protection between relays which are deployed between long distant. Any wireless technology that could be applied in a highly critical teleprotection application should have the following requirements:

- $\quad$ Ubiquitous coverage with a reliability of 99.999

- Sufficient redundancy between the end relays to avoid a single point of failure

- $\quad$ Latency varies based on the category, should be less 10 $\mathrm{ms}$ for category 2 applications, please see Table 3 [19] $\&[20]$.

- $\quad$ Support precise time synchronisation of less than $1 \mu \mathrm{s}$ between substation relays, subject to the service category and its criticality, teleprotection applications require very precise and high accurate time synchronisation. Precision Time Protocol (PTP) is a proper time synchronisation system that can be used between the relays with an accuracy better than $1 \mu \mathrm{s}$. Several other challenges may appear when looking at the time synchronisation.

- $\quad$ Security, authentication and integrity are needed while encryption is subject to application to assure the level of the needed security. Although power utility standards such as IEC 61850 outlines several end-to-end security principles to protect the data integrity, the deployed communication technology should provide additional measures against unauthorised access to the network.

Table 2 gives a high-level overview of different application requirements in terms of latency in smart grid automation

\begin{tabular}{|c|c|}
\hline \multicolumn{1}{|c|}{ Service } & Propagation Latency \\
\hline Category 1 & $\leq 6 \mathrm{~ms}$ \\
\hline Category 2 & $\leq 10 \mathrm{~ms}$ \\
\hline Category 3 & $\leq 30 \mathrm{~ms}$ \\
\hline
\end{tabular}


Table 3 5G Technical Specifications for Smart Grid interoperability) for $5 \mathrm{G}$ in utility should be fully understood

\begin{tabular}{|c|c|c|c|}
\hline Smart grid application & $\begin{array}{l}\text { Required } \\
\text { Latency }\end{array}$ & $\begin{array}{l}\text { Required } \\
\text { Data rates }\end{array}$ & Notes \\
\hline Teleprotection & $\begin{array}{l}{[5-30] \mathrm{ms}} \\
{[20]}\end{array}$ & $1 \mathrm{Mbps}$ & $\begin{array}{l}\text { Teleprotection requires low data rates - Teleprotection category } 1 \text { require } \\
\text { an end-to-end latency of } 5 \mathrm{~ms} \text {. }\end{array}$ \\
\hline Interlocking & $\begin{array}{l}\text { Less than } 100 \\
\text { ms }[21]\end{array}$ & $1 \mathrm{Mbps}$ & $\begin{array}{l}\text { The time delays to be set on the appropriate relays, which could require } \\
\text { end-to-end communication delay to be less than } 20 \mathrm{~ms} \text {. }\end{array}$ \\
\hline $\begin{array}{l}\text { HV Automation and } \\
\text { control }\end{array}$ & $1-20 \mathrm{~ms}[22]$ & $\begin{array}{l}1500 \mathrm{Kbps} \\
{[23]}\end{array}$ & $\begin{array}{l}\text { Dynamic control in the smart grid requires latency on the order of } 1 \mathrm{~ms} \text {. } \\
\text { - Cases such as wide-area situational awareness requires data rate on the } \\
\text { order of } 1500 \mathrm{Kbps} \text {. }\end{array}$ \\
\hline $\begin{array}{l}\text { Virtual Reality (VR) and } \\
\text { Augmented Reality } \\
\text { (AR) }\end{array}$ & $\begin{array}{l}1 \mathrm{~ms}[3 \mathrm{GPP} \\
\mathrm{TS} 23.203 \\
\text { Rel15] }\end{array}$ & 1 Gbps [24] & $\begin{array}{l}\text { Hi-resolution } 360 \circ \mathrm{VR} \text { (of } 12 \mathrm{~K} 3 \mathrm{D} \text { ) requires high rates on the order of } 1 \\
\text { Gbps with a latency of } 1 \mathrm{~ms} \text {. }\end{array}$ \\
\hline
\end{tabular}

It is good to mention that the exact requirements of the figures for latency presented above are application dependent and that all the services mentioned above should have a high level of availability. Other control applications that may require higher latency compared to the teleprotection requirements (i.e. communication of various signals such as breaker status and trip demand higher communication reliability). Secondary substation level control functions and microgrid applications are not considered in this study. Based on the above criteria, deciding whether $5 \mathrm{G}$ can be used for very critical applications such as teleprotection for power utility will be discussed in the remaining part of this paper.

\section{TELEPROTECTION CATEGORY 1 OVER RADIO COMMUNICATION}

So far, no radio technology has been used for teleprotection category 1 application, except microwave packet radio with a limited number of hops. As a test has been carried out at the PNDC to verify the performance of packet radio system for carrying power system current differential teleprotection applications and demonstrating the feasibility and suitability of migrating from existing TDM radio to packet radio. For the hardest test of meeting the 'propagation and asymmetrical latency', the results showed that the most stringent propagation latency requirement (i.e. $6 \mathrm{~ms}$ ) and asymmetrical latency requirement (i.e. $0.4 \mathrm{~ms}$ ) defined by ENA TS 48-6-7 could be satisfied using packet radio network for the only couple of hops. As it is good to mention that the challenges in terms of signal availability in rural areas and any lack of synchronisation between the microwave stations will impact on the findings.

As the $4 \mathrm{G}$ is already in the market, heavily deployed and used globally, also the associated cost of deploying such technology is less than the $5 \mathrm{G}$ and could make $4 \mathrm{G}$ more appropriate system to be applied in smart grid compared to $5 \mathrm{G}$. The business case and time to market will be covered later in the remaining sections of this paper. Many technical considerations (such as current connectivity, coverage and

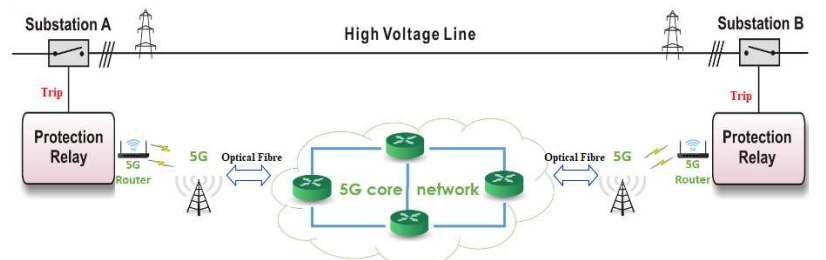

Figure 2 Teleprotection over a 5G Network before any $5 \mathrm{G}$ deployment in power networks as shown in the proposed 5G architecture for teleprotection, see Figure 2. Another service such as tele-interlocking will require delays of value lower than $<10 \mathrm{~ms}$, while other control applications between switches and controllers will need less than $<\sim 100$ ms.

\section{MAIN Challenges of 5G IN CRITICAL TIER 1 TELEPROTECTION}

\section{A. Fibre Optics are Needed}

Inputs from the PNDC's Mobile Network Operators (MNOs) tier 1 members [25], suggest plans to use optical fibre (where possible) in their (front/back/middle) haul network to enable the required synchronization needed to run the $5 \mathrm{G}$ properly. This means that the Distribution Network Operators (DNOs) will not be able to rely on $5 \mathrm{G}$ only to enable the required support and reliable connectivity for teleprotection applications without fibre.

\section{B. Synchronization is a Critical Challenge}

Although, 3GPP TS 38.133 standard stated the required phase synchronization accuracy measured at the Base Station (BS) antenna connectors to be better than $3 \mu \mathrm{s}$. Where, maximum phase/time difference of $3 \mu \mathrm{s}$ on the air interface between any end to end field device requires time error less than $\pm 1.5 \mu \mathrm{s}$. There will be a challenge in meeting the requirements of critical end applications such as teleprotection, as the question of how the synchronization information is transported wirelessly within and between the protection relays in an accurate/proper time will be a consideration.

Each hop in $5 \mathrm{G}$ front hall requires a point-to-point connection and accuracy of time source should be met in each $5 \mathrm{G}$ nodes. This will significantly increase the cost to enable teleprotection and there will be no guarantee that the highly critical end applications which will be run over $5 \mathrm{G}$ networks will have its required synchronization accuracy from the $5 \mathrm{G}$ networks. In collaboration with PNDC, the MNOs are currently exploring a cost-effective approach to enable such accuracy, precision, and resolution in $5 \mathrm{G}$ environment. The following are the main smart grid synchronization challenges in $5 \mathrm{G}$ :

- The main challenge is in the radio part (fronthaul) where accurate synchronisation needed for a protection relay could be affected by the weather and the signal propagation. $5 \mathrm{G}$ may not be sufficient to guarantee high accuracy source of synchronisation for tier 1 teleprotection applications. As the radio 
channel will transmit all of the traffic, it will also carry the accurate clock timestamps needed by the end application.

- Network congestion, time of the day could increase latency which causes a problem in synchronisation.

- While TDD is the most used, time synchronization is difficult to achieve unlike in FDD.

- Loss of GPS will create a problem in the $5 \mathrm{G}$ which in turn can cause a mal-operation in the communication between the relays.

- Accuracy requirements, where each component will add time error, more devices in the network will add more errors and could cause a problem.

- MNOs are aiming to rely on GPS, which could be easily affected by jamming and weather conditions which could transport error to the IED and reduce the reliability of the teleprotection performance.

\section{Interoperability and Signal Availability Challenge}

The compliance with the industrial and international standards (e.g. IEC 61850) ensures interoperability between the control networks of smart grids and the distributed intelligent devices. Any deployed technology for category 1 teleprotection should significantly boost interoperability in manufacturing by enabling IEDs to be connected. This may not be available in the coming few years as the legacy equipment along with the high cost of any new installations will be the main obstacles to see $5 \mathrm{G}$ in teleprotection category 1. Moreover, challenges in terms of 5G signal availability along with the propagation difficulties and the cost of reliable $5 \mathrm{G}$ technology in the rural areas make it difficult for $5 \mathrm{G}$ to enable the category 1 teleprotection. The above summary aligns with the statement from the Joint Radio Company white paper which was prepared for the Utilities Technology Council [26], which stated that "5G is unlikely to be able to meet an electricity utility's end-to-end latency requirement over a wide area" due to signal processing and coding times which will add more delay to the end to end latency. It is good to keep in mind that network delays and synchronization timings are two different problems. As the delay can cause problems to the time synchronisation, this could create an issue in the traffic or an unexpected event in mobile networks such as a natural disaster.

\section{Resilience Challenge}

The main concerns for resilient $5 \mathrm{G}$ in critical smart grid applications will lead to consideration of the following challenging scenarios in any future deployment for $5 \mathrm{G}$ in teleprotection application:

- The network performance during network congestion (some recent simulation - Efacec, Oeiras, Portugal [27] - shows that blocking signals often failed to arrive on time in a congested network, network slicing can be a solution to tackle that).

- Black start scenario (current installation at the MNO does not comply with the ENA specifications and requirements for the DNOs, where, enough backup power is needed to bring the grid back to operation as soon as possible).

- The availability of the signal in hard to reach areas is still a big challenge.

- $\quad$ Redundancy, can smart grid critical applications (i.e. teleprotection category 1 relies completely on wireless technology? This does not appear to be feasible under the current situation and the existing regulations and policies).

\section{SUMMARY OF MAIN 5G PROMISES AND CHALLENGES FOR PROTECTION APPLICATIONS}

Teleprotection is an essential part of a power grid infrastructure and using the $5 \mathrm{G}$ network for teleprotection functions will improve the smooth operation of the grid. Extending coverage enhancement to rural areas could become cost-effective by reducing the use of fibre and increasing the use of cellular networks. It will also allow for efficient fault detection, restoration, load balancing, and other remote operations through various teleprotection command schemes. When these processes are efficient and timely provisioned, they can impact the grid infrastructure and consumer services. For instance, fast disconnection of faulty sections of the grid reduces the energy surge and error propagation. All such uRLLC requirements can be achieved on the same mobile broadband of the $4 \mathrm{G}$ macro network which can be upgraded to $5 \mathrm{G}$ for line differential protection [28]. Permissive tripping, direct tripping, and blocking protection schemes as identified in [7] were considered based on reliability, speed of operation, security, local equipment permission, and the channel of communication. The protection requirements categories as defined in [12] specifies time synchronization of less than $20 \mu$ s between substations, $99.99 \%$ reliability, the latency of less than 10 ms, a disjoint redundant path between substations up to 50 $\mathrm{km}$ apart and secured using good authentication and encryption algorithms. 5G virtualization, network slicing, mobile edge computing and SDN concepts are all relevant for smart grid [11]. In substation protection, network slicing is used to isolate grid services and virtualization to increase network processing time.

The main challenges of $5 \mathrm{G}$ in utility sectors are scaling rural coverage, future-proofing, time to full deployment, reliability and security.

- $5 \mathrm{G}$ vs fibre for Category 1 teleprotection (right now sounds to be still early and very costly especially with the requirements for the services to be resilient for a black start when losing the power).

- $\quad 5 \mathrm{G}$ with URLLC feature and ubiquitous coverage could work with Category $2 \& 3$ (i.e. $>10 \mathrm{~ms}$ latency) but subject to availability and cost of any comprehensive deployment. So $5 \mathrm{G}$ could be used in critical teleprotection for power utility in Category 2, however, the number of hops should be considered. Also, recovery and resilience are needed for any critical teleptoection route. 
- Routable - GOOSE via 5G could open the door for applications such as fast tripping and high-speed blocking in Medium Voltages and more successful use cases (i.e. various control and protection applications via switches/reclosers with less $100 \mathrm{~m} . \mathrm{s}$ ).

- The limited coverage of small cells supported with uRLLC feature will create more hops to reach the endto-end connected relay with an ill increase in the endto-end delay.

Private network operators could tackle some of the above challenges and it can be used by the power utilities to help enable and optimise their critical business processes. As it is targeting specific service and applications, the power utility can go through this option to assure optimized, dedicated and secured network. Several reasons that utilities might want to operate a private network:

- The private network can be designed to operate in the absence of primary power, which could help in recovering any power outage quickly. An extended back-up power along with diverse and redundant routing of backhaul communications networks is crucial for any DNOs.

- The ability to deploy security techniques that are recommended by the regulator and comply with the industrial and international standards.

- Extend the coverage into more rural areas not served by the MNOs, where regular mobile network services are not available.

- Performance assurance via highly available and reliable networks and guarantee QoS for critical power applications

- Ensure that power utility technicians and engineers can communicate when repairing lines or restoring service after an outage.

\section{VIII.CONCLUSION}

This paper discussed whether 5G technology can be used for smart grid teleprotection critical applications. It has been presented that the requirements for the Category 1 applications with less than $6 \mathrm{~ms}$ may not be straight forward and satisfied in the coming years. where, another challenges such as $5 \mathrm{G}$ synchronisation, lack of $5 \mathrm{G}$ rural coverage and long delays caused by the number of hops along with the lack of backup power are the main constraints that stop $5 \mathrm{G}$ from being a reliable and cost-effective option to the teleprotection Category 1 . The success of $5 \mathrm{G}$ use cases in teleprotection is based on the cost to support uRLLC feature, this will require considering the security, guaranteed QoS, redundancy, and also adequate backup power.

\section{ACKNOWLEDGMENT}

The authors acknowledge the contributions of PNDC members (mainly, Scottish Power Energy Networks, Scottish and southern Electricity Networks UK power networks and Vodafone) and their valuable discussions over different meetings, workshops, research activities and knowledge exchange forum.

\section{REFERENCES}

[1]

S. Mojtaba Matinkhah and W. Shafik, "Smart Grid Empowered by 5G Technology," in 2019 Smart Gird Conference, SGC 2019, 2019.

[2] A. Hassebo, A. A. Mohamed, R. Dorsinville, and M. A. Ali, "5Gbased Converged Electric Power Grid and ICT Infrastructure," in IEEE 5G World Forum, 5GWF 2018 - Conference Proceedings, 2018, pp. 33-37.

[3] W. Zheng, K. Sun, X. Zhang, Q. Zhang, A. Israr, and Q. Yang, "Cellular Communication for Ubiquitous Internet of Things in Smart Grids: Present and Outlook," in Proceedings of the 32nd Chinese Control and Decision Conference, CCDC 2020, 2020, pp. 5592-5596.

[4] A. E. Labrador Rivas and T. Abrão, "Faults in smart grid systems: Monitoring, detection and classification," Electr. Power Syst. Res., vol. 189, p. 106602, Dec. 2020.

N. Dorsch, F. Kurtz, and C. Wietfeld, "On the economic benefits of software-defined networking and network slicing for smart grid communications," NETNOMICS Econ. Res. Electron. Netw., vol. 19, no. 1-2, Oct. 2018.

[6] M. Patzold, "5G Unlocks Its Power for Global Mobile Connectivity [Mobile Radio]," IEEE Veh. Technol. Mag., vol. 15, no. 2, pp. 5-11, Jun. 2020.

[7] B. Appasani, J. B. R. Maddikara, and D. K. Mohanta, "Standards and Communication Systems in Smart Grid," Springer, Singapore, 2019, pp. 283-327.

[8] S. R. S, T. Dragičević, P. Siano, and S. R. S. Prabaharan, "Future Generation 5G Wireless Networks for Smart Grid: A Comprehensive Review," Energies, vol. 12, no. 11, p. 2140, Jun. 2019.

[9] H. C. Leligou, T. Zahariadis, L. Sarakis, E. Tsampasis, A. Voulkidis, and T. E. Velivassaki, "Smart Grid: A demanding use case for 5G technologies," in 2018 IEEE International Conference on Pervasive Computing and Communications Workshops, PerCom Workshops 2018, 2018, pp. 215-220.

[10] S. Borenius, J. Costa-Requena, M. Lehtonen, and R. Kantola, "Providing network time protocol based timing for smart grid measurement and control devices in 5G networks," in 2019 IEEE International Conference on Communications, Control, and Computing Technologies for Smart Grids, SmartGridComm 2019, 2019.

[11] T. A. Zerihun and B. E. Helvik, "Dependability of Smart Distribution Grid Protection Using 5G," in Proceedings - 2019 3rd International Conference on Smart Grid and Smart Cities, ICSGSC 2019, 2019, pp. 51-59.

[12] G. Bag, L. Thrybom, and P. Hovila, "Challenges and opportunities of 5G in power grids," in CIRED - Open Access Proceedings Journal, 2017, vol. 2017, no. 1, pp. 2145-2148.

[13] 3GPP, "TS $123501-$ V15.2.0 - 5G; System Architecture for the 5G System (3GPP TS 23.501 version 15.2.0 Release 15),” 2018.

[14] 3GPP, "TR $121915 \quad$ - V15.0.0 - Digital cellular telecommunications system (Phase 2+) (GSM); Universal Mobile Telecommunications System (UMTS); LTE; 5G; Release description; Release 15 (3GPP TR 21.915 version 15.0.0 Release 15)," 2019.

[15] K. Ghanem, R. Asif, S. Ugwuanyi, and J. Irvine, "Bandwidth and security requirements for smart grid," in IEEE PES Innovative 
Smart Grid Technologies Conference Europe, 2020, vol. 2020October, pp. 36-40.

[16] K. Ghanem, I. Abdulhadi, A. Kazerooni, and C. McGookin, "Communication requirements for future secondary substations to enable DSO functions," CIRED Work., Sep. 2020.

[17] S. Meng, Z. Wang, M. Tang, S. Wu, and X. Li, "Integration Application of 5G and Smart Grid," in 2019 11th International Conference on Wireless Communications and Signal Processing, WCSP 2019, 2019.

[18] X. Xia et al., "A Survey on 5g network slicing enabling the smart grid," in Proceedings of the International Conference on Parallel and Distributed Systems - ICPADS, 2019, vol. 2019-Decem, pp. 911-916.

[19] ENA, "TS 48-6-7 (Issue2) - Communication Services for Teleprotection Systems," 2013.

[20] ENA, "TS 48-6-9 (Issue 1) - Communication Bearers Used for the Provision of Teleprotection Channels," 2010.

[21] ENA, "Engineering Recommendation G99 Issue 1 - Amendment 1 - Requirements for the connection of generation equipment in parallel with public distribution networks on or after 27 April 2019," May 2018.

[22] P. Schulz et al., "Latency Critical IoT Applications in 5G: Perspective on the Design of Radio Interface and Network Architecture," IEEE Communications Magazine, vol. 55, no. 2. Institute of Electrical and Electronics Engineers Inc., pp. 70-78, 01-Feb-2017.

[23] Amitava Ghosh, "5G mmWave Revolution and New Radio Recording ," IEEE Future Networks, 20-Sep-2017. [Online]. Available: https:/thewirelessuniversity.com/wpcontent/uploads/2019/04/NAI1_Ghosh_5G_NR_5GmmWave_W ebinar_IEEE_Nokia_09_20_2017_final.pdf. [Accessed: 20-Jan2021].

[24] USA Department of Energy, "Communications Requirements of Smart Grid Technologies | Department of Energy," Oct. .

[25] PNDC, "University of Strathclyde Power Networks Demonstration Centre (PNDC) Memberships," 21-Jan-2021. [Online]. Available: https://pndc.co.uk/memberships/. [Accessed: 21-Jan-2021].

[26] JRC, "Cutting Through the Hype: 5G and Its Potential Impacts on Electric Utilities," Mar. 2019.

[27] A. Aleixo et al., "Resilient 5G technologies optimized for power grid protection solutions using IEC 61850 time-critical communications," in 15th International Conference on Developments in Power System Protection (DPSP 2020), 2020, pp. 055 (6 pp.)-055 (6 pp.).

[28] G. Wikstrom et al., "Wireless Protection of Power Grids over a 5G Network," in 2019 IEEE PES GTD Grand International Conference and Exposition Asia, GTD Asia 2019, 2019, pp. 976981. 